\title{
Elliptical tracks: evidence for superluminal electrons?
}

\author{
KeIth A. Fredericks \\ Restframe Labs, New York, NY USA \\ keith@restframe.com
}

\begin{abstract}
In the literature of Low-Energy Nuclear Reactions (LENR), particle tracks in photographic emulsions (and other materials) associated with certain electrical discharges have been reported. Some Russian and French researchers have considered these particles to be magnetic monopoles. These tracks correspond directly to tracks created with a simple uniform exposure to photons without an electrical discharge source. This simpler method of producing tracks supports a comprehensive exploration of particle track properties. Out of 750 exposures with this method, elliptical particle tracks were detected, 22 of which were compared to Bohr-Sommerfeld electron orbits. Ellipses fitted to the tracks were found to have quantized semi-major axis sizes with ratios of $\simeq n^{2} / \alpha^{2}$ to corresponding Bohr-Sommerfeld hydrogen ellipses. This prompts inquiry relevant to magnetic monopoles due to the $n^{2} / \alpha^{2}$ force difference between magnetic charge and electric charge using the Schwinger quantization condition. A model using analogy with the electron indicates that the elliptical tracks could be created by a bound magnetically charged particle with mass $m_{m}=1.45 \times 10^{-3} \mathrm{eV} / \mathrm{c}^{2}$, yet with superluminal velocities. Using a modified extended relativity model, $m_{m}$ becomes the relativistic mass of a superluminal electron, with $m_{0}=5.11 \times 10^{5} \mathrm{eV} / \mathrm{c}^{2}$, the fine structure constant becomes a mass ratio and charge quantization is the result of two states of the electron.
\end{abstract}

Keywords: particle tracks, monopoles, tachyons, superluminal, faster-than-light, elliptical orbits, photographic emulsion, Kepler orbits, LENR, strange radiation

\section{Introduction}

In this paper evidence is reported for quantized elliptical tracks in photographic emulsions with sizes expected of bound magnetic monopoles, yet requiring velocities greater than the speed of light, indicating particles with superluminal electric charge.

Preliminary measurement of the first recognized elliptical track detection showed a size difference, to within a few percent of $137^{2} n^{2} \simeq n^{2} / \alpha^{2}$ larger than an $n=7$ Bohr-Sommerfeld hydrogen electron orbit, $\alpha^{-2}$ being the difference in force between electrons and magnetic monopoles using Schwinger quantization, i.e. $g=2 g_{D}$ (Eq. (2)). This runs contrary to current expectations suggesting magnetic monopole bound states with smaller (e.g. $7.2 \times$ $10^{-18} \mathrm{~m}$ ) orbit sizes [1] than bound electrons in hydrogen. 
Additional experiments provided a collection of elliptical track candidates that were fitted to ellipses. The resulting track fits are quantized according to semi-major axis size, (see Fig. 1) and therefore energy, (see subsec. 5.3) according to $E^{(n)}=h \nu^{(n)}$, indicating a new particle exhibiting properties of magnetic monopoles. Analogy with the electron and orbit sizes suggest faster-than-light velocities.

Analysis of the results leads to a new model of extended relativity restricted to bound states using a scale transformation rather than the usual superluminal Lorentz transformation (SLT), leading to the fine structure constant as a mass ratio and a new proposed explanation for charge quantization.

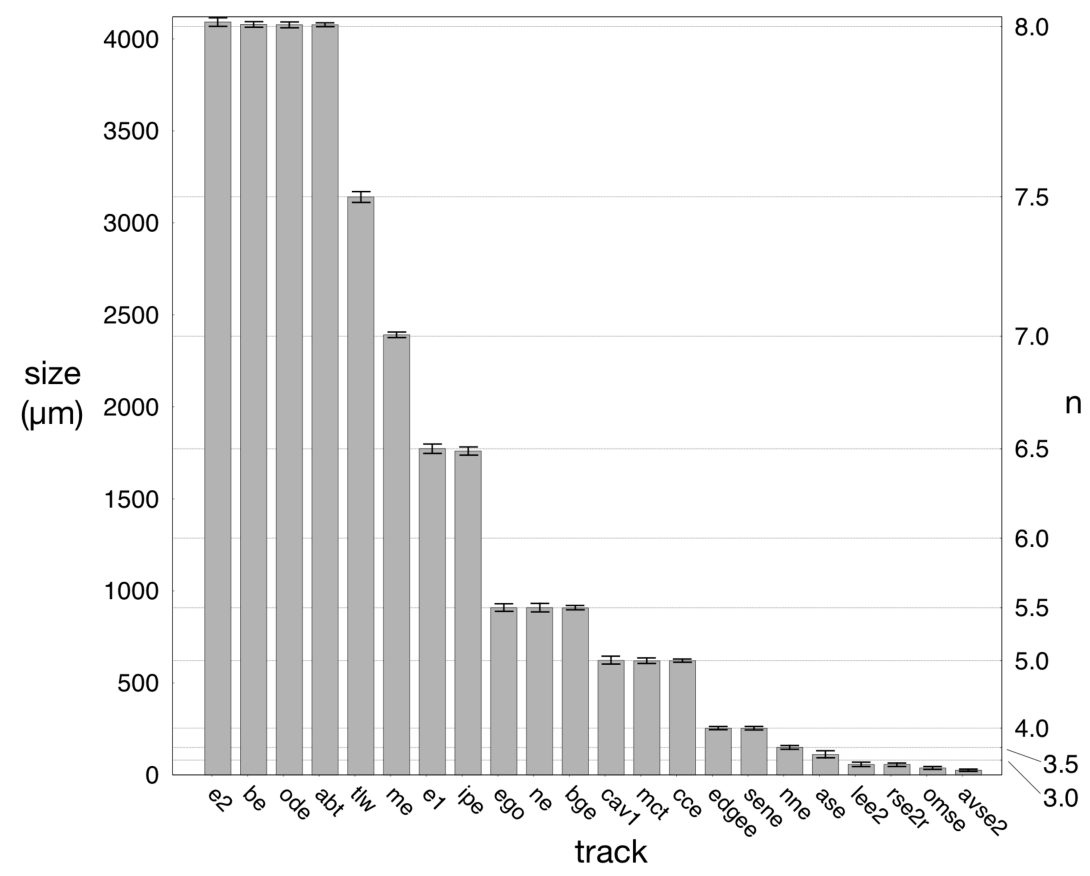

Figure 1: Quantized ellipse semi-major axis sizes. Ellipses between $n=8$ and $n=3.5$ are shown to be quantized as half integer values. Ellipses less than $n=3.5$ are quantized by quarter integer values. Error bars represent the combined standard uncertainty of the semi-major axis size.

\section{Elliptical tracks}

In earlier work [2], properties of new and unusual tracks in photographic emulsions were analyzed and classified. The source of the tracks was unidentified. Analysis showed track configurations clearly distinct from those observed before in nuclear track studies [3], but directly corresponding to tracks from certain studies including:

1. exposure of emulsions during bombardment by low-energy ions in electric explosion of metallic wires and thin foils [4-7], 
2. exposure of emulsions during low-energy discharges in water and excitation of betadecay products by a magnetic field [8],

3. exposure of emulsions and Pd electrodes during glow discharge plasma processes [9],

4. exposure of special semiconductor layers during the supercompression of solid targets using electron beams [10], and,

5. exposures of photographic emulsions, CR-39 (with and without etching), PMMA, and glass during picosecond laser irradiation of electrodes [11].

Ivoilov [8] called this unknown source a control background.

Using a simple brief uniform amplifying exposure of photons on photographic emulsions exposed to the environment (as in e.g. a cosmic ray study) duplicates virtually all of the track types presented in the list of studies above. This technique is called photon amplification.

The advantages of photon amplification are:

1. Tracks can be readily seen in the plastic layer as well as the emulsion layer of photographic films.

2. Compared to electric discharge sources, simple conditions of the exposure to light bypasses the need for elaborate laboratory setups and hence more data can be gathered (hundreds of exposures) and the phenomenon can be studied more deeply.

3. Amplification or sensitization of the photographic emulsion due to the uniform photon exposure increases the sensitivity of the photographic emulsion to photons and charged particles.

4. Detector sensitivity is re-zeroed at a higher level by the uniform photon exposure so as to react to tearing down as well as building up of latent image. White tracks are detected as well as black tracks.

5. The light sensitive volume element of the photographic emulsion registers track images of usual timelike partcles, which travel through space and, (if they were to exist and are detectable), spacelike particles, which would travel through time, since the detector is continually active until the time of development.

This study is concerned primarily with tracks, in photographic emulsions coated on a plastic base, produced during (or after) brief exposures to light. These exposures to light produce a uniform background density on development. Tracks in emulsion are registered against this background density in addition to tracks registered in the plastic base.

Supplemental uniform exposures of photons followed by or combined with imagewise fluxes of particles (traditionally photons) can enhance the sensitivity of photographic emulsions [12].

Giving the supplemental exposure prior to the imagewise exposure is called pre-exposure [13] or pre-flashing. Giving the supplemental exposure during the same time interval is called concurrent photon amplification [14]. These techniques were used experimentally by photographic scientists and practically in astronomy and in autoradiography, but (outside of the present study) apparently never used with emulsions for detection of charged particle tracks 


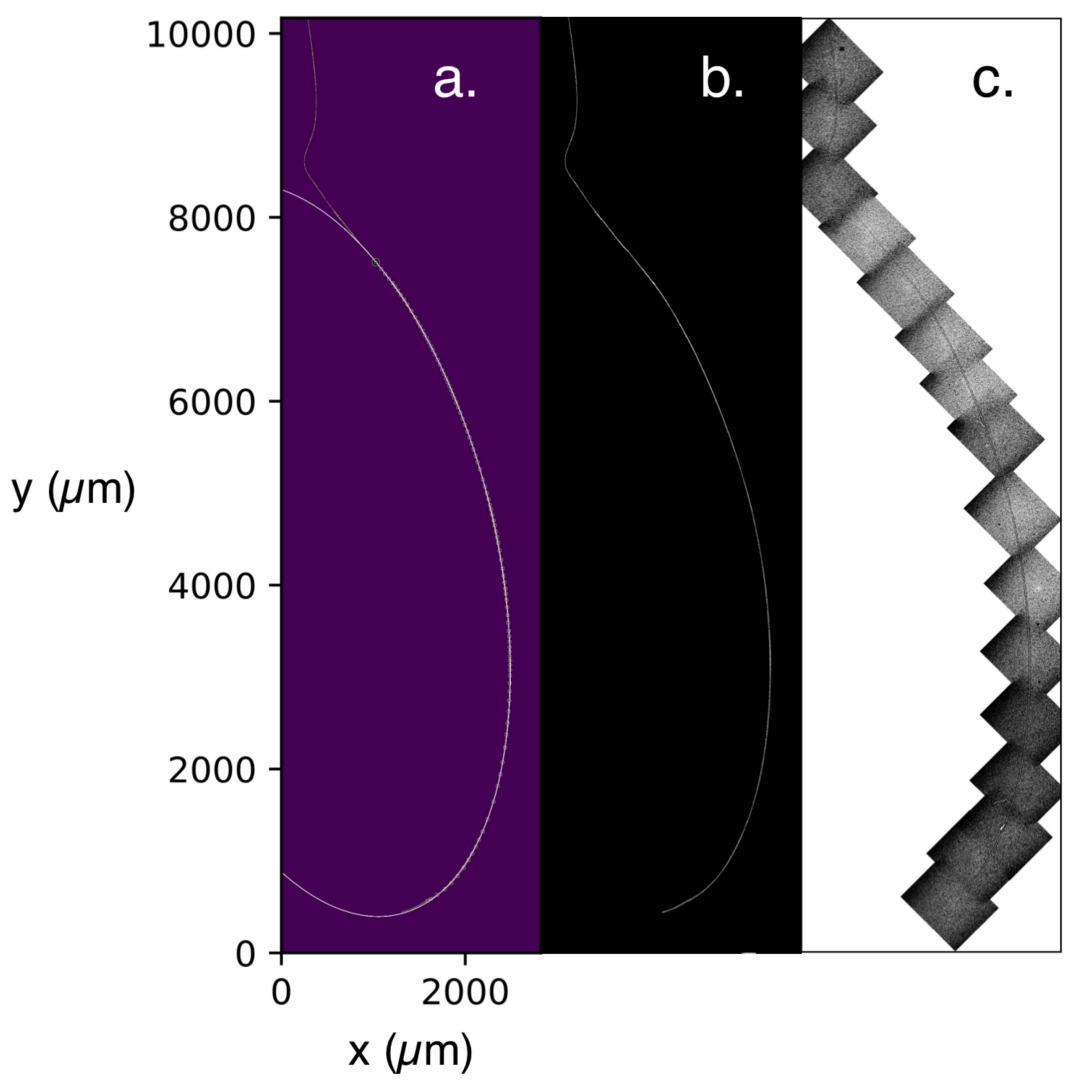

Figure 2: Track be (see also Table 1). The semi-major axis size of $a_{m}=4078.6 \pm 14.6 \mu \mathrm{m}$ is related to the semi-major axis size of an $n=8$ electron in hydrogen, $a_{e}$ by $a_{m} / 137^{2} n^{2} \simeq a_{e}$. a.) Least-squares fit of $x, y$ track coordinates to ellipse overlayed on processed track image. b.) Track after image processing and background eradication. c.) Photo montage of track.

(nuclear track studies). One of the critical questions to be answered is how is it possible that a particle, responsible for these tracks, has so far evaded detection, especially with a history of perhaps 150 years of the scientific use of photographic emulsions. Here are some possible explanations:

1. Since the tracks are generally microscopic effects, they would not normally be recognized in non-uniform (standard photography) backgrounds and, if seen, they would be easy to dismiss or ignore as a processing errors, light leaks or other artifacts.

2. Since (apparently) no nuclear track studies, that is where emulsions were scanned by greater than $\sim 250 x$ magnification, used supplementary photon exposures as amplification, the probability of these new types of tracks remaining unrecognized would be increased.

3. In the case where no supplemental photon exposure is required to create a baseline background of clear tracks (in plastic base or gelatin), and where magnification greater 
than $\sim 250 x$ was used to scan emulsions, these tracks may well have not been the object of the search or not of interest, being clear rather than black, or they may have been mistaken for something else like surface damage ( $c f$. Ref. [7]).

4. In the case where no supplemental photon exposure is used and where black tracks, as were reported here, were registered on the film and the film was scanned with greater than $\sim 250 x$ magnification, then it is possible that these tracks were treated as unidentified and never looked at in a deeper way.

5. In general, the rate of detection/recognition and the flux (see subsec. 3.4) was reduced and more capricious compared to objects being studied, decreasing the chance of recognition.

For electrically (magnetically) charged particles, it is expected that magnetic fields (electric fields) applied perpendicularly to the plane of particle motion will produce helical curvature and it is expected that electric fields (magnetic fields) applied transversely to the plane of particle motion will produce parabolic curvature. In [2] the possibility of parabolic curvature in magnetic fields for these tracks was incorrectly asserted. In a large number of experiments using applied magnetic fields from $\sim 6.0 \times 10^{-4}$ to $\sim 0.7 \mathrm{~T}$, and electric fields from $\sim 1 \times 10^{5}$ to $2 \times 10^{5} \mathrm{~V} / \mathrm{m}$, these particles have not, so far, appeared to follow helical or parabolic paths. Rather, they appear to move in other unpredictable ways.

During these experiments, however, repeatable evidence has been found for tracks with elliptical curvature occurring in the presence or absence of applied electric and/or magnetic fields. Detection of elliptical curvature is probably the most significant breakthrough in the study of these tracks, providing the possibility to compute, under certain assumptions, via purely geometrical considerations, properties of particles capable of creating these tracks.

\section{Experimental}

\subsection{Technique}

Experiments between May 2014 and December 2017 used uniform photon amplification of films with applied magnetic and electric fields in various configurations. Results were scanned for curved tracks.

Film types Arista Ortho Litho 3.0 or Rollei Ortho 25 were exposed both in $10.2 \mathrm{~cm} \mathrm{x}$ $12.7 \mathrm{~cm}$ and $3 \mathrm{~cm} \times 12.7 \mathrm{~cm}$ sheets. Three types of apparatus comprised of LEDs, applied magnetic fields and applied electric fields were used:

1. Anti-Helmholtz coil of radius $6.3 \mathrm{~cm}$ with 10 turns each of 14 AWG wire, running at $3.0 \mathrm{~A}$ with a computed field of $0.0 \mathrm{~A} / \mathrm{m}$ at the center and computed magnetic flux density at the center of $0.0 \mathrm{~T}$. The coils were aligned parallel to the horizontal vector of the earth's magnetic field pointing north. A thin cylinder NdFeB magnet, $r=9 \mathrm{~mm}$, $h=3 \mathrm{~mm}$, with remanence of $B_{r}=1.32 \mathrm{~T}$ was placed in the center of the film in the center of the coils, south pole facing down and 2 white LEDs at a $3-6 \mathrm{~cm}$ distance were turned on simultaneously using an Arduino Uno with a 5 s delay followed by a 50ms $2000 \mathrm{~ms}$ exposure. 
2. Solenoid with film on external stage with LED illumination as in 1 . The axis of the solenoid of 410 turns and length $0.123 \mathrm{~m}$ with $12 \mathrm{VDC}$ and $3 \mathrm{~A}$ was oriented $(+/-)$ at $0^{\circ}$ to $68^{\circ}$ to the film plane generating $1.26 \times 10^{-2} \mathrm{~T}$ and a 0 to $7200 \mathrm{VDC}$ E-field applied transversely using $\mathrm{Cu}$ electrodes with $3 \mathrm{~cm}$ separation. A yoked $\mathrm{NdFeB}$ magnet with flux density of $0.5 \mathrm{~T}$ on the center line between poles perpendicular to the $x, y$ plane of the film was opposite to the solenoid.

3. Solenoid with film on internal stage with LED illumination as in 1 . The axis of a solenoid of 2703 turns and length $0.757 \mathrm{~m}$ with $12 \mathrm{VDC}$ and $3 \mathrm{~A}$ was oriented (+/-) at $0^{\circ}$ to $68^{\circ}$ to horizontal generating $1.35 \times 10^{-2} \mathrm{~T}$ with the film plane slightly tilted with respect to the solenoid axis.

LEDs, magnetic fields and electric fields were all controlled with an Arduino Uno.

Various white LED lamps were used for the uniform amplifying exposure. These LEDs have a typical luminous intensity of $1250 \pm 15 \% \operatorname{mcd}$.

Film was handled only using vinyl gloves. Under recommended safelight illumination, the film was marked to indicate orientation and placed onto the exposure stage, normally in a north-south direction.

Film was then developed for 90s immediately after exposure. Development was stopped with a water bath, normally fixed and dried. Using a Leitz Metalloplan microscope with a large stage, developed film was scanned at low magnification. At higher magnifications, selected tracks were measured using an eyepiece reticle calibrated with a stage micrometer and photographed using plano objectives and a calibrated Peltier-cooled 12 MP Jenoptik ProgRes C14 camera. Selected images were then aligned in mosaics, processed, measured and analyzed using Image J microscopy software and a set of custom track processing routines written in Python.

\subsection{Observations}

Experiments were performed with various electric and magnetic field configurations and various levels of light amplification of $>750$ exposures comprising a total photosensitive area of $>2.85 \mathrm{~m}^{2}$. No repeatable curvature resulting from the application of electric or magnetic fields was observed. It is possible that the conditions for electric or magnetic curvature of the particles was outside of the applied field strengths. It is also possible that applied fields played a role in the production of elliptical tracks. 102 of these tracks were candidates for elliptical curvature analysis. About $20 \%$ of the track candidates showed excellent unambiguous fits to ellipses.

The elliptical track type is a partial ellipse (ellipse segment) and can be described as a comma track. This type of curvature is observed either in standalone tracks or tracks indicating a particle decay event or a series of decays. If not a standalone track, ellipses commonly appear at one end of a track (See Fig. 5) or as an intermediary component of a compound track, which may be a series of partial ellipses. Partial ellipses also commonly 
occur in pairs, multiples and swarms as do non-ellipse tracks ( $c f$. [2] and see subsec. 7.1). Partial ellipses can be registered as black tracks or white tracks in the emulsion layer or clear tracks in the plastic base. On examination with 400x magnification, along with the darkening or bleaching of $\mathrm{AgBr}$ grains, many tracks show physical formation of voids, damage and/or ionization along $\sim 90 \%$ of the track indicating particle traversal cutting a channel on the surface or directly through the $\mathrm{AgBr} /$ gelatin layer and/or plastic base of the film. Daviau, et al. [7], using confocal and scanning electron microscopes, make the same point about particles cutting a groove or tunneling through layers of the emulsion and base. Due to the length and smooth curvature of the tracks, this indicates a particle detection with unusual characteristics and appears to rule out known charged particles.

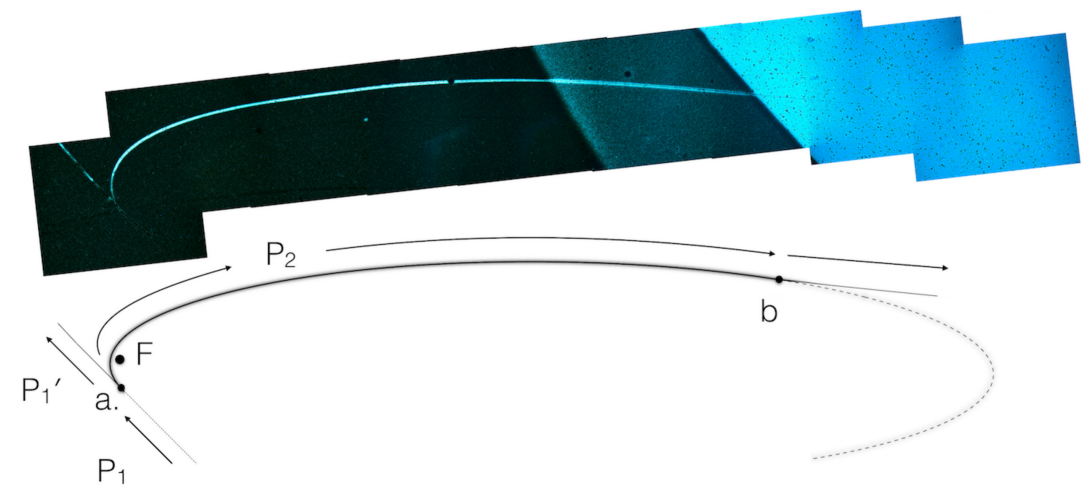

Figure 3: Track me, $n=7$. Top.) Semi-major axis size $a=$ $2391.6 \pm 16.1 \mu \mathrm{m}$. Ten tile photomosaic at 160x using a Leitz PL 16x objective. See Fig. 4 for closeup of track. Bottom.) Capture into and escape from an elliptical orbit. 1.) initial particle, $P_{1}$, trajectory. 2.) at point a. particle decays into $P_{1}^{\prime}$, continuing on initial trajectory and $P_{2}$, which is captured into an elliptical orbit. 3.) at point b. particle escapes from the elliptical orbit.

In each case the elliptical tracks can be viewed as classical capture into and escape from a Kepler elliptical orbit in the plane of the film. In Fig. 3 (Bottom), the initial particle, $P_{1}$ decays into $P_{1}^{\prime}$ and $P_{2} . P_{2}$ is then captured into and subsequently escapes from an elliptical orbit.

In analogy with the gravitational case, we assume orbital capture of (charged) particle $P_{2}$ with mass $m$ is possible due to a massive (charged) particle at the focus, $F$, of the ellipse in Fig. 3 (Bottom) with mass $M>>m$ (and opposite charge). It is not clear why the particle escapes from the elliptical orbit.

Elliptical trajectories where $n>3$ are observed to escape before aphelion. When $n<3$, some ellipses are observed to continue on until almost complete.

\subsection{Measurement}

After creating a photomosaic of the track at high magnification, image processing is used to eradicate the background and $x, y$ coordinates are extracted. Entry and exit points are 


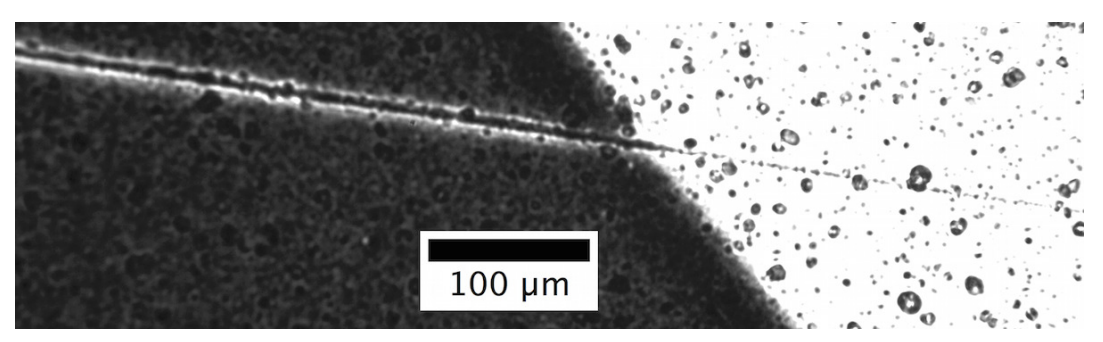

Figure 4: Detail of the $n=7$ track showing track as blackened grains in dark area and as voids in plastic or gelatin in clear area. Note the conventional pattern similar to an ionizing particle in an emulsion ( $c f$. periodic tracks in Ref. [[2]] and references therein).

determined by test hand-fitting followed by automated fitting using a progressive least-squares algorithm.

The procedure for measuring these tracks is as follows:

1. Scan entire film for elliptical tracks using a microscope with $25 \mathrm{x}$ magnification

2. Locate elliptical track candidate and change to higher $(\geq 160 \mathrm{x})$ magnification.

3. Locate, measure and record fiducial points near to track.

4. Photograph the track in sections

5. Reconstruct track using mosaic (photomontage) software

6. Scale $x, y$ track coordinates to $x, y$ image coordinates

7. Threshold track image (image processing)

8. Eradicate background (image processing)

9. Get track center $x, y$ coordinates (image processing)

10. Determine ellipse entry and exit points

11. Fit ellipse to $x, y$ coordinates

12. Report result as size of ellipse axes \pm error. See Table 1

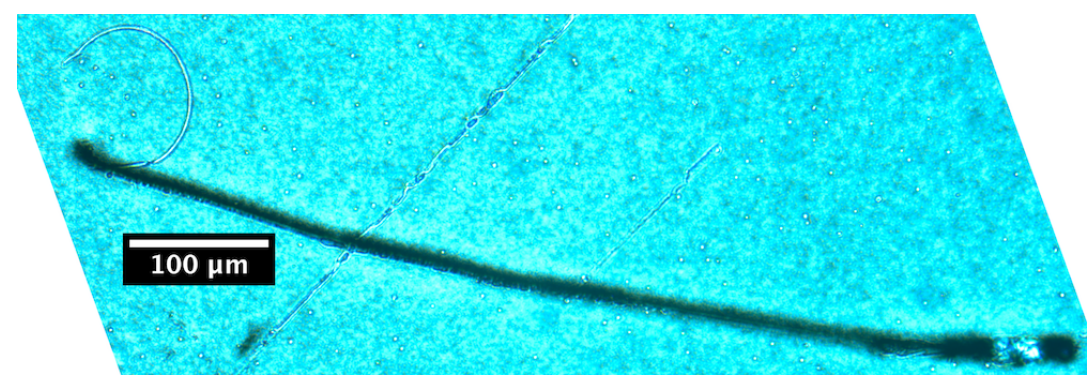

Figure 5: Track lee2, $n=2.75$ showing ellipse at "end" of track. "Clear" ellipse emerges as if ejected from straight track with both clear and darkened components. Photographed at 160x using a Leitz PL 16x objective. 


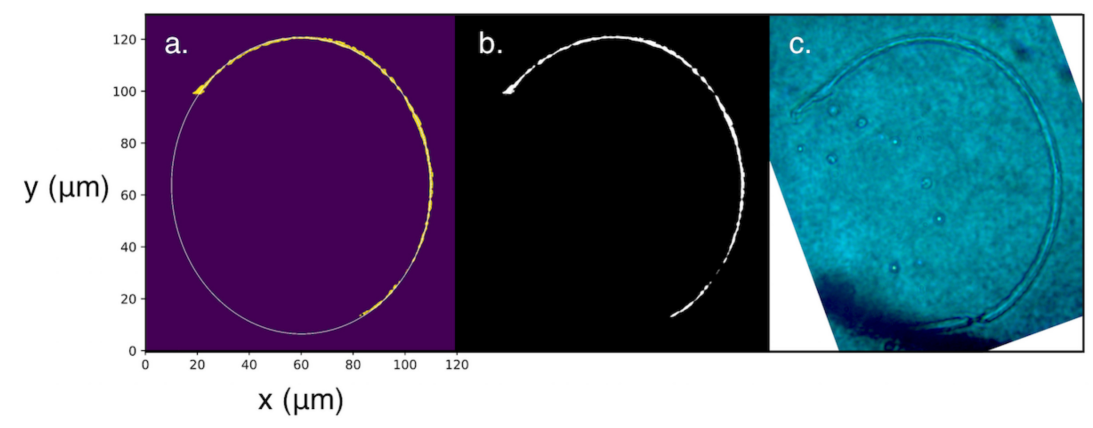

Figure 6: Track lee2, $n=2.75$. a.) Ellipse fitted to track, semimajor axis size, $a=56.9 \pm 13.0 \mu \mathrm{m}$. b.) After processing and background eradication. c.) Photo at 1000x using Leitz PL 100x objective.

\subsection{Particle flux}

Based on an average exposure and processing time of 180 s with a detector square area of $\sim 3.8 \times 10^{-3} \mathrm{~m}^{2}$ and an estimate of five general tracks per exposure, these particles have a differential flux of $\sim 1.2 \mathrm{~m}^{-2} \mathrm{~s}^{-1} \mathrm{sr}^{-1}$. This compares with $1 \mathrm{~m}^{-2} \mathrm{~s}^{-1} \mathrm{sr}^{-1}$ for $500 \mathrm{MeV}$ electrons and $1.1 \mathrm{~m}^{-2} \mathrm{~s}^{-1} \mathrm{sr}^{-1}$ for $500 \mathrm{MeV}$ photons at sea level [15]. About 750 exposures with these parameters were carried out. Of these, 22 final ellipses were found, putting the total ellipse track flux at $\sim 1.8 \times 10^{-6} \mathrm{~m}^{-2} \mathrm{~s}^{-1}$.

\section{Framework for analysis}

\section{1. $1 / r^{2}$ force tracks in film}

A $1 / r^{2}$ central force and an orbiting particle are required to create an elliptical particle track in a nuclear track detector.

It is not possible, however, with any known charged particle to record a significant part of an elliptical particle track or any track with appreciable smooth curvature with attainable magnetic fields in photographic emulsions. In this sense, these tracks are more like tracks in bubble chambers, that is, cutting through the relatively high density photographic emulsion like a hot knife through butter.

For known charged particles, scattering of the particle obscures any curvature and the most that can be expected of measurements of curvature occurring entirely within photographic emulsions due to applied magnetic fields is to find the sign of charge [16].

The central force involved is active over a range from $\sim 2.54 \times 10^{-5} \mathrm{~m}$ to $4.07 \times 10^{-3} \mathrm{~m}$. The strong force, weak force and gravity are negligible at these distances. Electrostatic central force is within the range with the largest known electron orbits of order $n \sim 1000$ in Rydberg atoms with radius $10^{-4} \mathrm{~m}$ [17], but is ruled out by the inability of tracks of known charged particles to register smooth curvature in emulsions. The remaining possibility is magnetostatic central force, i.e. a magnetic charge orbiting an opposite magnetic charge. 
Table 1: Ellipse semi-major, $a$, and semi-minor, $b$, axis measurements for each track along with associated quantum number.

\begin{tabular}{lrrc}
\hline \hline track & $a(\mu m)$ & $b(\mu m)$ & $n$ \\
\hline e2 & $4091.7 \pm 22.5$ & $2241.3 \pm 22.5$ & 8.00 \\
be & $4078.6 \pm 14.6$ & $1996.0 \pm 14.6$ & 8.00 \\
ode & $4077.0 \pm 16.1$ & $1129.4 \pm 16.1$ & 8.00 \\
abt & $4076.6 \pm 10.3$ & $1053.6 \pm 10.3$ & 8.00 \\
tlw & $3141.0 \pm 30.5$ & $1474.4 \pm 30.5$ & 7.50 \\
me & $2391.6 \pm 16.1$ & $549.3 \pm 16.1$ & 7.00 \\
e1 & $1773.2 \pm 24.9$ & $1100.5 \pm 24.9$ & 6.50 \\
ipe & $1760.0 \pm 23.1$ & $615.3 \pm 23.1$ & 6.50 \\
ego & $908.9 \pm 21.8$ & $505.8 \pm 21.8$ & 5.50 \\
ne & $909.0 \pm 25.7$ & $367.2 \pm 25.7$ & 5.50 \\
bge & $907.5 \pm 11.6$ & $284.3 \pm 11.6$ & 5.50 \\
cav1 & $622.1 \pm 21.1$ & $300.7 \pm 21.1$ & 5.00 \\
mct & $619.9 \pm 16.2$ & $192.7 \pm 16.2$ & 5.00 \\
cce & $621.1 \pm 7.3$ & $173.8 \pm 7.3$ & 5.00 \\
edgee & $254.2 \pm 7.4$ & $104.8 \pm 7.4$ & 4.00 \\
sene & $253.6 \pm 9.8$ & $202.2 \pm 9.8$ & 4.00 \\
nne & $149.8 \pm 13.1$ & $83.0 \pm 13.1$ & 3.50 \\
ase & $111.1 \pm 20.6$ & $72.1 \pm 20.6$ & 3.25 \\
lee2 & $56.9 \pm 13.0$ & $49.8 \pm 13.0$ & 2.75 \\
rse2 & $56.7 \pm 9.5$ & $31.5 \pm 9.5$ & 2.75 \\
omse & $38.1 \pm 6.2$ & $36.0 \pm 6.2$ & 2.50 \\
avse2 & $25.6 \pm 6.4$ & $15.9 \pm 6.4$ & 2.25 \\
\hline \hline
\end{tabular}

\subsection{Geometric analysis}

Ordinarily calibration with a known particle track type is done in nuclear emulsions for particle identification via particle energy loss. Since only geometric analysis of the tracks is being done in this case, this type of calibration is not required.

\subsection{Magnetic monopoles}

Dirac [18] showed that quantized magnetic charges (hereafter monopoles) could explain the quantization of electric charge and bring symmetry to Maxwell's equations. But the symmetry is not complete since the value of magnetic charge does not equal the value of electric charge.

The Dirac quantization condition [18],

$$
g_{D}^{(n)}=\frac{e c n}{2 \alpha}, \quad n=(0, \pm 1, \pm 2, \pm 3, \ldots)
$$




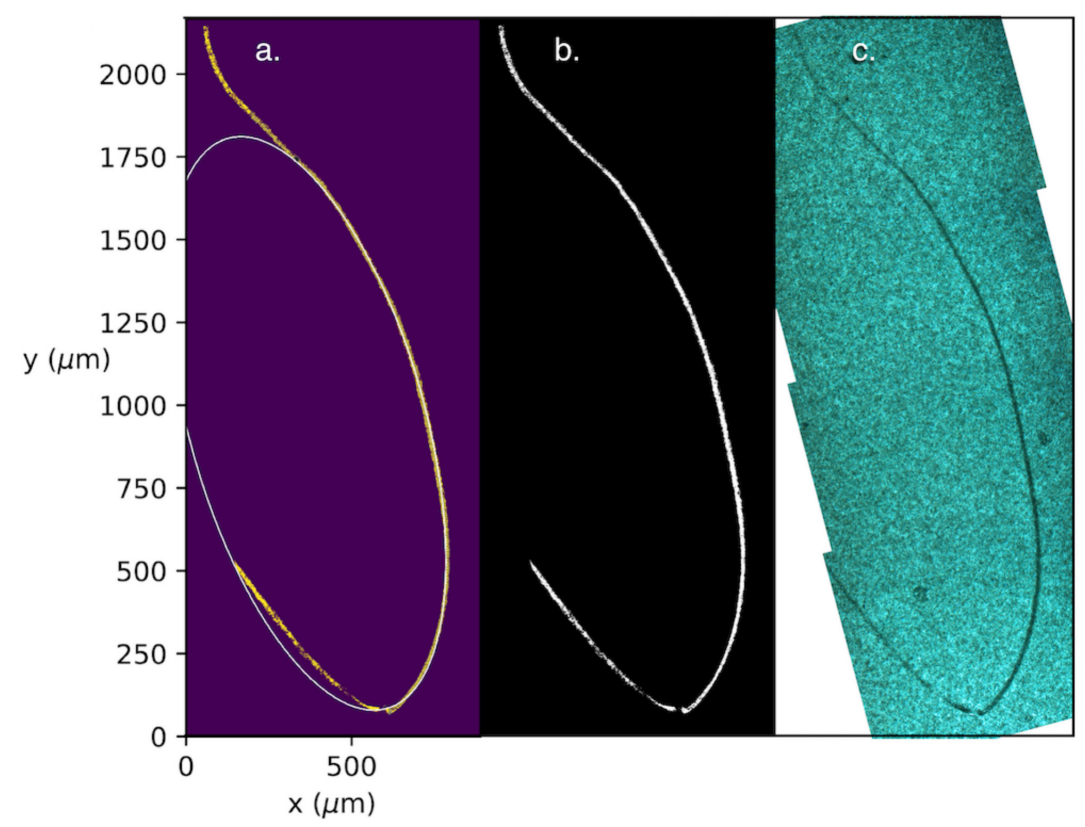

Figure 7: Track $n e, n=5.5$. a.) Ellipse fitted to track, semimajor axis size, $a=909.0 \pm 25.7 \mu m$. b.) Track after processing and background eradication. c.) Mosaic of track photographed with Leitz PL 16x objective.

where $\alpha=k_{e} e^{2} / \hbar c$ and $k_{e} \equiv\left(4 \pi \varepsilon_{0}\right)^{-1}$ with $n=2$ gives

$$
g=2 g_{D}=\frac{e c}{\alpha}
$$

and is equivalent to the $n=1$ Schwinger Quantization $\left(g=2 g_{D}\right)$ condition [19]. The Schwinger formulation removed Dirac's thin solenoid and gained rotational symmetry.

Using Eq. (1) with $n=1$, the force between north and south magnetic poles is $\varepsilon_{0} \mu_{0}\left(g_{D} / e\right)^{2}$ or $68.5^{2}$ times stronger than the force between electric charges and using Eq. (2), the force difference or ratio between magnetic and electric charges is

$$
\varepsilon_{0} \mu_{0}\left(\frac{g}{e}\right)^{2}=\frac{n^{2}}{\alpha^{2}}, \quad\left(g=2 g_{D}\right)
$$

or, with $n=1, \alpha^{-2}=137^{2}$.

A classical monopole (if it exists) should move in an elliptical orbit around an oppositely charged pole at one focus and that pole should have a mass much greater than the orbiting pole.

Based on analogy with the electron and using $g=2 g_{D}$, the magnetic coupling constant

$$
\alpha_{m}=\alpha^{-1}=k_{m} \frac{g^{2}}{\hbar c}, \quad\left(k_{m} \equiv \mu_{0} / 4 \pi\right)
$$


where subscripts of $m$ indicate magnetic, is just the reciprocal of the fine structure constant, $\alpha$.

\section{Particle properties}

Semi-major axes of the fitted ellipses, $a_{m}$, differ from the semi-major axes, $a_{e}$, of corresponding Bohr-Sommerfeld ellipses for hydrogen by $\simeq 137^{2} n^{2}$. Using $g=2 g_{D}$,

$$
137^{2} n^{2} \simeq \frac{a_{m}^{(n)}}{a_{e}^{(n)}}=\frac{n^{2}}{\alpha^{2}},
$$

where subscripts of $e$ stand for the electron and superscripts of $(n)$ denote principal quantum numbers. Semi-major axis sizes and quantum numbers for the magnetic ellipses are determined using

$$
a_{m}^{(n)}=a_{e}^{(n)} \frac{n^{2}}{\alpha^{2}}, \quad(\alpha=e c / g),
$$

where $\alpha$ is the Schwinger quantization from (2). See Table 2. These are compared with the collection of fitted ellipses. In Fig. 1 measured ellipse sizes are shown to be quantized by half integer values from $n=8$ to $n=3.5$. Below $n=3.5$, ellipse sizes appear to be quantized by quarter integer values.

\subsection{Monopole mass}

All orbits including the Bohr radius follow the same relation as Eq. (5), so that with $n=1$,

$$
\frac{a_{0 m}}{a_{0 e}}=\frac{1}{\alpha^{2}} .
$$

Using the formula for Bohr radius, $a_{0 e}=\hbar / m_{e} c \alpha$, the analogous $a_{0 m}=\hbar / m_{m} c \alpha_{m}$ and Eq. (7), monopole mass can be written as

$$
m_{m}=\frac{m_{e} \alpha^{3}}{\alpha_{m}}
$$

and from Eq. (4), $\alpha_{m}=\alpha^{-1}$, so

$$
m_{m}=m_{e} \alpha^{4}=1.45 \times 10^{-3} \mathrm{eV} / \mathrm{c}^{2},
$$

which leads to the fine structure constant, $\alpha=\left(m_{m} / m_{e}\right)^{1 / 4}$, as a mass ratio.

This value agrees with our independent prediction of monopole mass [20] based on $g=2 g_{D}$. See subsec. 6.4. 
Table 2: Ellipse semi-major axis size, $a_{m}$, energy, $E$, velocity, $v$ and momentum, $p$ for $n=\{1-8\}$. Mass, $m_{m}=1.45 \times 10^{-3} \mathrm{eV} / \mathrm{c}^{2}$.

\begin{tabular}{ccccc}
\hline \hline$n$ & $a_{m}(\mathrm{~m})$ & $E(\mathrm{eV})$ & $v(\mathrm{~m} / \mathrm{s})$ & $p(\mathrm{eV} / \mathrm{c})$ \\
\hline 1 & $9.93 \times 10^{-7}$ & $1.36 \times 10^{1}$ & $4.11 \times 10^{10}$ & $1.99 \times 10^{-1}$ \\
2 & $1.59 \times 10^{-5}$ & $8.51 \times 10^{-1}$ & $8.22 \times 10^{10}$ & $2.48 \times 10^{-2}$ \\
3 & $8.04 \times 10^{-5}$ & $1.68 \times 10^{-1}$ & $1.23 \times 10^{11}$ & $7.36 \times 10^{-3}$ \\
4 & $2.54 \times 10^{-4}$ & $5.32 \times 10^{-2}$ & $1.64 \times 10^{11}$ & $3.11 \times 10^{-3}$ \\
5 & $6.21 \times 10^{-4}$ & $2.18 \times 10^{-2}$ & $2.05 \times 10^{11}$ & $1.59 \times 10^{-3}$ \\
6 & $1.29 \times 10^{-3}$ & $1.05 \times 10^{-2}$ & $2.46 \times 10^{11}$ & $9.20 \times 10^{-4}$ \\
7 & $2.38 \times 10^{-3}$ & $5.67 \times 10^{-3}$ & $2.88 \times 10^{11}$ & $5.80 \times 10^{-4}$ \\
8 & $4.07 \times 10^{-3}$ & $3.32 \times 10^{-3}$ & $3.29 \times 10^{11}$ & $3.88 \times 10^{-4}$ \\
\hline \hline
\end{tabular}

\subsection{Monopole velocity}

The ground state orbital velocity of the electron in hydrogen is $v_{0 e}=k_{e} e^{2} / \hbar=c \alpha$ and the analogous ground state orbital velocity of the bound monopole

$$
v_{0 m}=k_{m} \frac{g^{2}}{\hbar}=c \alpha^{-1}
$$

where $v_{0 m}>c$, once seen as required yet unphysical [21] is postulated as required, physical and aptly symmetric [20] with the electron, i.e. $v_{0 e}=c \alpha$ below $c$ and $v_{0 m}=c \alpha^{-1}$ above $c$ (see Table 3).

\subsection{Quantized energy levels}

For the electron in hydrogen, energy is quantized according to

$$
E_{e}^{(n)}=-k_{e} \frac{e^{2}}{2 a_{0 e} n^{2}}=\frac{E_{0 e}}{n^{2}}=h \nu^{(n)}
$$

and for the bound monopole,

$$
E_{m}^{(n)}=-k_{m} \frac{g^{2}}{2 a_{0 m} n^{4}}=\frac{E_{0 m}}{n^{4}}=h \nu^{(n)} .
$$

Note the $n^{-2}$ vs. $n^{-4}$ difference between the electron and monopole.

\subsection{Kepler period}

Using $g=2 g_{D}$, the Kepler period for an electron in hydrogen and the monopole in a magnetic hydrogen analog,

$$
\tau_{m}^{(n)}=\frac{2 \pi a_{0 m} n^{3}}{v_{0 m}}=\frac{2 \pi a_{0 e} n^{3}}{v_{0 e}}=\tau_{e}^{(n)},
$$

are equal. 


\section{A new superluminal transformation}

\subsection{Extended relativity}

The concept of special relativity extended to superluminal velocities was perhaps best put by Corben [22] where our world is bifurcated at the speed of light with one part (our regular world) moving at $v^{2}<c^{2}$ and the other part moving at $v^{2}>c^{2}$ relative to us. The two parts of the world are symmetric since what we observe as bradyons from the $v^{2}<c^{2}$ part will be observed as tachyons from the $v^{2}>c^{2}$ part and vice-versa. Observations by physicists in each world are defined as equivalent, but with rest frames at different relative velocities.

A cornerstone of the work done by Recami and others to extend special relativity to superluminal velocities is the superluminal Lorentz transformation or SLT [23, 24] that connects observations made in a $v^{2}>c^{2}$ frame with observations made in the $v^{2}<c^{2}$ frame. A superluminal particle property observed by a subluminal observer needs to be transformed to correspond to something that we would normally observe.

Using an SLT, length, time, momentum, energy and mass can all be transformed between $v^{2}<c^{2}$ and $v^{2}>c^{2}$ frames, timelike vectors are transformed into spacelike vectors and vice-versa. Velocity is transformed simply as $\beta \rightarrow 1 / \beta$ where $\beta=v / c$.

In this framework, an electron from a $v^{2}>c^{2}$ frame seen by an observer in a $v^{2}<c^{2}$ frame is interpreted as an electron with superluminal Lorentz-transformed properties and magnetic charge of $g=-e c$ (bringing a more complete symmetry to Maxwell's equations). Slower-than-light monopoles are not predicted in this theory.

The collinear bi-dimensional $(x, t)$ SLT works without problems, but, when extended to four dimensions $(x, y, z, t)$, it leads to imaginary quantities for certain measurements of $v^{2}>$ $c^{2}$ objects and problems of how to interpret these physically. It is possible to use a sixdimensional space for the SLT, but there can be difficulties with the physical interpretation of two additional time dimensions.

If length is contracted using a collinear bi-dimensional transcendent [23] SLT along the $\mathrm{x}$ axis and further (isotropic scale) contracted by an EM shift of $\alpha$ in accordance with the Schwinger quantization condition where $a_{m}^{(n)}=x, a_{e}^{(n)}=x^{\prime}, b_{m}^{(n)}=y, b_{e}^{(n)}=y^{\prime}$,

$$
\left\{\begin{array}{l}
a_{m}^{(n)}(\alpha \gamma)=a_{e}^{(n)} \\
b_{m}^{(n)}(\alpha)=b_{e}^{(n)}
\end{array} \quad\left(\gamma=\left(\beta^{2}-1\right)^{-1 / 2}, \quad \beta>1\right)\right.
$$

Using theoretical $n=8, \ell=2$ ellipse data, ellipse size, orientation and eccentricity $(\mathscr{E}=$ $\left.0.95 \rightarrow \mathscr{E}^{\prime}=0.99\right)$ radically changes as a result of this hybrid SLT.

Using Recami's multidimensional approach, the SLT simply inverts the quadratic form sign [25] of an object and the shape of the monopole ellipse becomes

$$
0 \geq-\frac{x^{2}}{a_{m}^{(n) 2}}+\frac{y^{2}}{b_{m}^{(n) 2}} \geq-1
$$


consisting of the area between an on-central-axis conic section,

$$
\frac{y^{2}}{b_{m}^{(n) 2}}=\frac{x^{2}}{a_{m}^{(n) 2}},
$$

and a hyperbola,

$$
\frac{y^{2}}{b_{m}^{(n) 2}}=\frac{x^{2}}{a_{m}^{(n) 2}}-1,
$$

greatly distorted from the original ellipse with eccentricity, $\mathscr{E}^{\prime}>1$, as shown in Fig. 8. A real

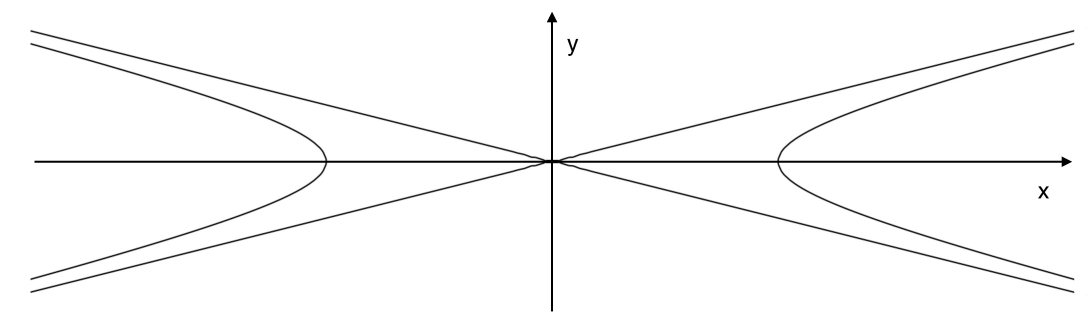

Figure 8: Recami type SLT of a theoretical $n=8, \ell=2$ monopole ellipse.

(vs. complex) SLT by Rajput, et.al [26] results in an ellipse with the same semi-major axis size, same eccentricity, $\mathscr{E}=\mathscr{E}^{\prime}$ and an axis swap from $x$ to $t$.

The elliptical particle tracks we observe, (do they share geometrical properties of superluminal electrons?), do not share the same semi-major axis size as their (presumed) subluminal counterparts and do not appear to be distorted. That is, they appear to be scaled instead of stretched.

\subsection{The Coulomb flip}

If measured eccentricity corresponds to pure Bohr-Sommerfeld eccentricity, then, due to the $\alpha^{-2}$ size change, the transformation of ellipses in general needs to be an isotropic scale transformation instead of a translation along one axis.

The ratio of measured eccentricity, $\mathscr{E}_{\mu}$ to Bohr-Sommerfeld eccentricity, $\mathscr{E}_{\tau}$

$$
\frac{\mathscr{E}_{\mu}}{\mathscr{E}_{\tau}}=\frac{\left(1-b^{2} / a^{2}\right)^{1 / 2}}{n^{-1}\left(n^{2}-\ell(\ell+1)\right)^{1 / 2}}
$$

as a ratio of averages over the 22 track values, $\left\langle\mathscr{E}_{\mu}\right\rangle /\left\langle\mathscr{E}_{\tau}\right\rangle=0.99$, and measured eccentricity corresponds well to Bohr-Sommerfeld electron orbit eccentricity. This implies that the superluminal transformation, for this restricted case of bound particles, should be a scale transformation.

The required transformation is just the force ratio, from Eq. (3), a scale ratio, dilatation $\left(\alpha^{-2}\right)$ or contraction $\left(\alpha^{2}\right)$ between magnetic and electric force strengths, which could be called the Coulomb flip. 
The one-dimensional basis Coulomb flips from $\beta>1$ to $\beta<1$ are

$$
\begin{array}{rrl}
\alpha_{\mathrm{EM}}: & \alpha_{m} & \left(\alpha^{2}\right)=\alpha_{e}, \\
a_{0}: & a_{0 m} & \left(\alpha^{2}\right)=a_{0 e}, \\
v_{0}: & \alpha_{m} c & \left(\alpha^{2}\right)=\alpha_{e} c \\
\text { and } q^{2}: & k_{m} g^{2} & \left(\alpha^{2}\right)=k_{e} e^{2} .
\end{array}
$$

Combining the basis transformations, Eqs. (19b), (19c), and (19d) and assuming $\hbar$ is Coulomb flip invariant, transformations for momentum, energy and mass are

$$
\begin{aligned}
& p_{m}=\frac{\hbar}{a_{0 m}} \quad \underset{\left(\alpha^{2}\right)}{\stackrel{(1)}{\longrightarrow}} \frac{\hbar}{a_{0 e}}=p_{e} \\
& E_{m}=-\frac{k_{m} g^{2}}{2 a_{0 m}} \quad \stackrel{\left(\alpha^{2}\right)}{\underset{\left(\alpha^{2}\right)}{\longrightarrow}}-\frac{k_{e} e^{2}}{2 a_{0 e}}=E_{e} \\
& m_{m}=\frac{\hbar}{a_{0 m} \alpha_{m} c} \quad \underset{\left(\alpha^{2} \alpha^{2}\right)}{\stackrel{(1)}{a_{0 e} \alpha_{e} c}}=m_{e}
\end{aligned}
$$

and the monopole and electron properties can be transformed into one another using Coulomb flips with $g=2 g_{D}$. In the ground state, time, in Eq. (13) and energy in Eq. (20b) remain invariant.

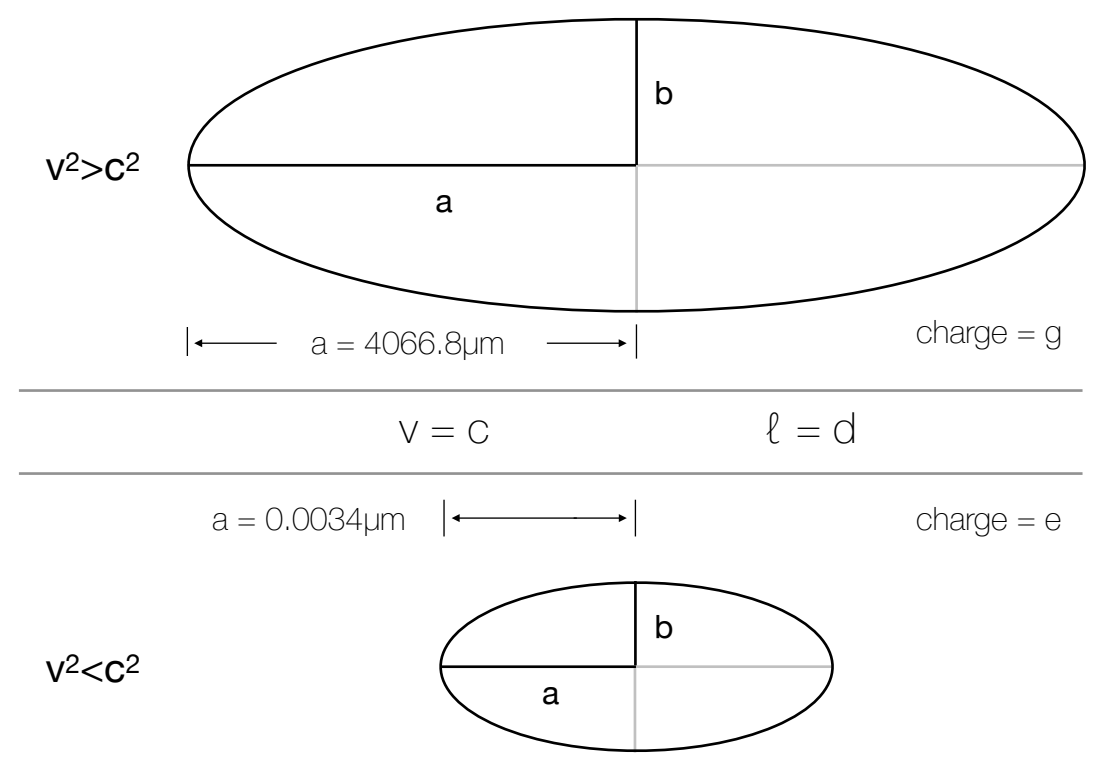

Figure 9: How a $v^{2}<c^{2}$ observer sees a $v^{2}<c^{2}$ electron orbit and an $n=8, l=2 v^{2}>c^{2}$ monopole orbit. The $v^{2}>c^{2}$ orbit is transformed to the $v^{2}<c^{2}$ orbit using the 2-dimensional Coulomb flip Eq. (21) (Not to scale).

The 2-dimensional Coulomb flip scales the standard equation of the monopole ellipse 
(upper ellipse in Fig. 9), by the factor $\left(n^{2} \alpha^{-2}\right)^{2}$,

$$
\left(\frac{n^{2}}{\alpha^{2}}\right)^{2}\left(\frac{x^{2}}{a_{m}^{(n) 2}}+\frac{y^{2}}{b_{m}^{(n) 2}}\right)=1,
$$

to the electron ellipse (lower ellipse in Fig. 9).

Since measurements made on the $v^{2}<c^{2}$ and $v^{2}>c^{2}$ sides of the world use $(x, y, z)$ quantities all scaled by the same real factor, $\left(n^{2} \alpha^{-2}\right)$, extending Eq. (21) to 3 dimensions, imaginary quantities do not enter the equations as they do in the determination of lengths orthogonal to the boost axis in the usual SLT. This scale transformation, resulting from interpretation of experiments, may, as a side-effect, solve the imaginary quantities problem [27] of SLTs.

The relativistic scale transformation could be termed a Lorentz zoom as opposed to a Lorentz boost. Relativistic scale transformations have been investigated by Nottale [28].

\subsection{Equivalence of charges}

Even though a superluminal particle's observed (by a subluminal observer) charge state is magnetic, it should possess exactly the same amount of charge in its own rest frame (the $v^{2}<c^{2}$ frame) where the charge state is electric. This is to say that $v^{2}>c^{2}$ electric charge is observed by normal subluminal observers to be magnetic charge and that subluminal magnetic charge does not exist [29, 30].

As noted earlier, equivalence of charge along with considerations of special relativity prompted Recami, et.al. to set $g=-e c$ and abandon the Dirac quantization condition with respect to magnetic monopoles. In light of $g=2 g_{D}$ it is possible to reconsider this issue.

The basis Coulomb flip for charge in Eq. (19d) shows that, after transformation, the magnetic charge is equivalent to the electric charge in a type III frame observed from a type I frame. Solving for $g$ in $(19 \mathrm{~d})$ with $k_{m} / k_{e}=1 / c^{2}$ yields $g=e c / \alpha$, which is the Schwinger quantization condition. So the Coulomb flip for charge is just the Schwinger quantization condition.

\subsection{Fundamental lengths}

In an analysis of the Dirac quantization condition, Datta [21], found a new hierarchy of fundamental monopole lengths, $a_{0 m}, \chi_{c m}, r_{0 m}$, (Bohr radius, Compton wavelength and classical 'electron' radius) and pointed out that monopole bound states require superluminal ground state orbital velocities, but rejected the superluminal velocities as unphysical.

With acceptance of the required superluminal velocities and $g=2 g_{D}$, a new hierarchy of fundamental lengths and corresponding velocities [20] (see Table 3) reproduces the extended relativity spacetime structure, but, in the restricted case of bound states, and due to the experimental result of Eq.(18), requires Coulomb scale transformations between subluminal and superluminal sides rather than SLTs. 
Table 3: Velocity and length sequence alignment [20].

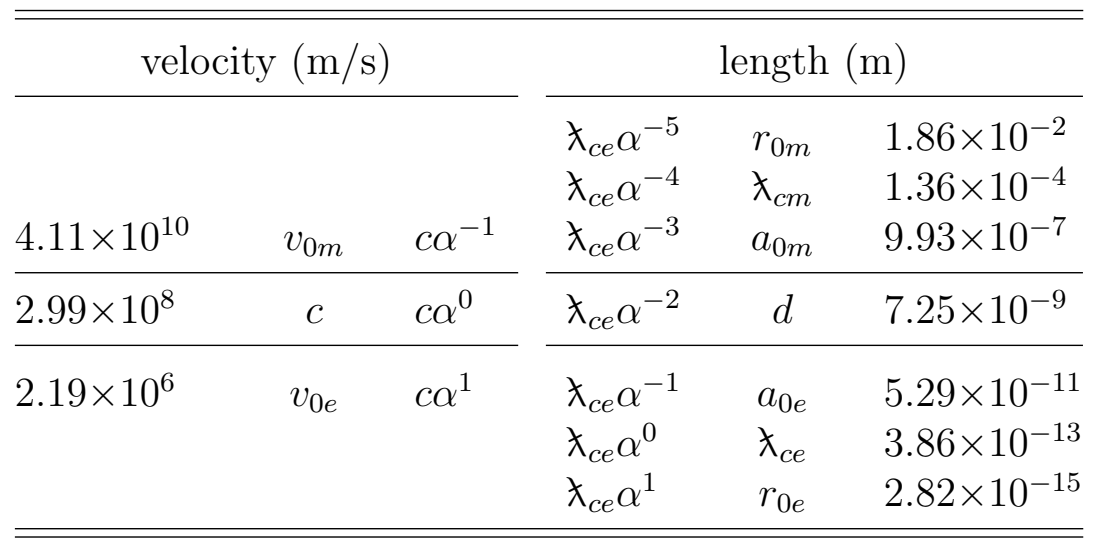

\section{Discussion}

These particles have been a mystery from the first known detection in 1979 (see Ref. [2]), using the photon amplification technique, to the experiments by Urutskoev and coworkers in 2000 and the various other experiments mentioned above in the ensuing years.

\subsection{Earlier findings}

The analyses of these experiments up to now have been based on certain observed particle properties such as the length of tracks, the curving of the tracks, the energy required to create a track in a certain material, etc.

Overall there has been agreement in the conjecture that these tracks are caused by monopoles [2, 4-8, 10, 11, 20, 31-33]. Until now we have known about certain particle properties:

1. Periodicity, visible in some (but not all) tracks, clearly indicates regular oscillation of the particle (system) causing the track [2, 4, 5, 7, 9, 11, 31-33]. Orderly helical-like tracks as well as complex periodic tracks have been observed.

2. Penetration, is apparent as many of the tracks possess very long track lengths, up to and more than $\sim 7 \mathrm{~cm}$ through photographic emulsions and other materials $[2,4-7,32]$.

3. Random motion, where the track undergoes many large-angle deflections like Brownian motion $[2,7,31]$.

4. Correlation of tracks $[2,8,11,31,34]$ indicates a common origin of particles that are likely entangled. This also includes correlated random motion tracks and swarms [2].

5. Central force evident from conformation of multiple correlated particle tracks $[2,7,8$, 31, 32]. Track geometry of correlated tracks indicates coordinated response arising from a central force.

6. Tracks in various materials, i.e. plastic, gelatin, metals, semiconductors and glass [2, 911] gives information about the energy of these particles.

7. Large angles of curvature or abrupt changes of directions of tracks [2, 8, 32]. may give information about the mass and charge of particles. 
8. White tracks in addition to black tracks indicate a tearing down of the latent image. These tracks can only be a result of exposure after or during the supplemental photon exposure, thus constraining the exposure time to the elapsed time after photon exposure till start of development. These tracks have only been observed in Fredericks [2]. The $\mathrm{AgBr}$ emulsion, re-zeroed at a higher level of sensitivity, able to respond in 2 directions, may be exactly the type of detector Terletskii [35] suggested as necessary to detect a negative energy particle.

Skvortsov has made mass estimates in a range of $\sim 10^{-3} \mathrm{eV} / \mathrm{c}^{2}[36]$ and $\sim 10^{-2} \mathrm{eV} / \mathrm{c}^{2}[11]$, based on the peak-to-peak amplitude of a periodic (clear) track in CR-39 (etched for 20 min. in an alkali solution). Mass shown in Eq. (9), $1.45 \times 10^{-3} \mathrm{eV} / \mathrm{c}^{2}$, is within this range.

\subsection{New findings}

The series of experiments culminating in detection of elliptical curvature of new tracks in emulsions and the subsequent analysis brings a new critical set of particle properties:

1. Exposures with and without applied electric and/or magnetic fields show nearly identical results in terms of track curvature. Most tracks are observed as curving one way and then the other or as long straight tracks. These particles (or composite particle systems) appear to behave as if neutral or the applied electric and magnetic fields are not in the right range to repeatably affect the particles.

2. Partial elliptical tracks show quantized semi-major axis sizes $a_{m}^{(n)}, n^{2} \alpha^{-2}$ larger than Bohr-Sommerfeld hydrogen with quantized energy levels $E^{(n)}=h \nu^{(n)}$.

3. Tracks show decay events, some of which are elliptical tracks emitted from a central track (see Fig. 10), showing two distinct track types.

4. Ground state velocity based on the elliptical tracks and analogy with the electron is $\alpha^{-2}$ greater than the electron ground state velocity in hydrogen, and therefore superluminal.

5. Coulomb flips of the new particle momentum, energy and mass match with a superluminal electron with $m_{0}=5.11 \times 10^{5} \mathrm{eV} / \mathrm{c}^{2}$.

6. The Coulomb flip of Eq. (19a) constitutes a single EM coupling constant, $\alpha_{\mathrm{EM}}$, which inverts with velocity

$$
\alpha_{E M}=\left\{\begin{array}{ll}
\alpha, & \text { if } \quad \beta<1 \\
\alpha^{-1}, & \text { if } \quad \beta>1
\end{array} .\right.
$$

7. Mass, $m_{m}$, based on the elliptical tracks and analogy with the electron, which is the relativistic mass of the electron, is $m_{e} \alpha^{4}$ smaller than the electron rest mass, giving a mass ratio equal to the fine structure constant from Eq. (9), $\alpha=\left(m_{m} / m_{e}\right)^{1 / 4}$.

8. Charge quantization based on special relativity, i.e Dirac's cause of quantization is the presence of magnetic charge, which is satisfied by the corresponding superluminal charge state of the electron, which to the subluminal electron is indistinguishable from magnetic charge. 


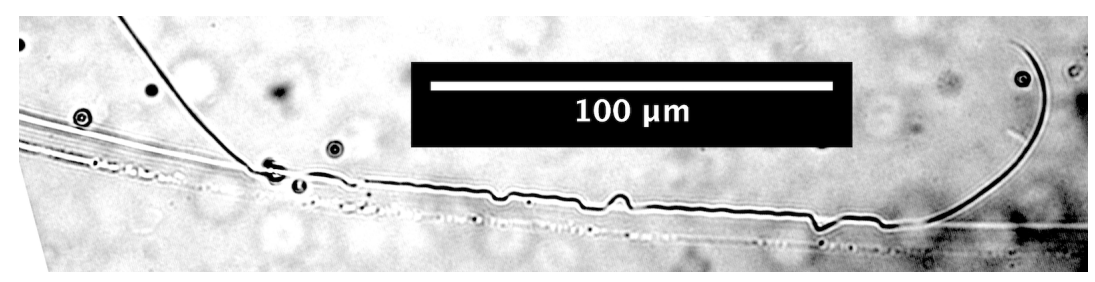

Figure 10: Track rse2, $n=2.75$ showing elliptical track decay event. Photo at 400x using Leitz PL 40x objective.

The key point is item 5. that, using the Coulomb flip, the particle tracks correspond directly to the geometry of electrons from a superluminal frame.

Application of electric and magnetic fields during exposures that create these tracks shows no hard evidence of affecting main track curvature. This seems to be contradictory since only charged particles should be recorded and if recorded should respond to magnetic and/or electric fields. However, the particle (or composite particle system) behaves as if neutral. Decay events, where particle tracks emerge from a main track, however, do exhibit repeatable curvature, may be susceptible to EM fields, and, as mentioned in item 3, show two specific particle types. Note that Daviau and coworkers [7] earlier referred to this decay as branching.

According to the Heisenberg uncertainty principle, our elliptical orbits should be smeared or diffuse, not sharp tracks. This question is beyond the current scope but the subject of an upcoming investigation.

\subsection{Theoretical approaches}

Georges Lochak $[32,33]$ has investigated these tracks and has looked specifically at the tracks produced in experiments by Urutskoev et al. [4, 5], Ivoilov [8, 37], Daviau et al. [7], Bardout et al. [38] and Priem et al. [6, 39] and suggests that these are light leptonic monopoles that are magnetically excited neutrinos. Lochak believes that these particles are linked to composite particles following De Broglie's theory of light [33]. Writing the equations for a nonlinear massive monopole, Lochak shows (as an aside) how tachyon states appear and that these states exist also for the nonlinear neutrino. Lochak cites Recami and Mignani in relation to this hypothesis. He also believes, based on work by Urutskoev and coworkers and Ivoilov, that these particles are extremely numerous.

Independent of the particle track data, Stumpf [40] elaborates on Lochak's ideas of the light leptonic magnetic monopole and composite particles on the basis of Urutskoev, et al.'s $[4,5]$ use of discharges in water as the source of low-energy nuclear reactions. Stumpf is in agreement with Lochak regarding the magnetically excited neutrino hypothesis and looks to extend the standard model to include these types of particles.

In the track images where a decay is taking place, i.e. a particle is seen flying off at an angle to a main trajectory, one can assert the idea of two or more particles traveling together, but then separating when a certain energetic conditions are met. The composite particle system concept may well fit with the physical evidence seen in these types of tracks. 


\subsection{The Coulomb lens}

The Coulomb flip likely provides a superluminal transformation without the introduction of imaginary quantities, allowing switching between electric and magnetic worlds and transforming all of the ellipses in the study from monopole to electron orbits. This would mean that superluminal objects could provide a portal of magnification, analogous to gravitational lensing, into our own world, that electron orbits could be examined in a way never before available, like looking at partial atomic electron orbits using the $\alpha^{-2} \mathrm{x}\left(\sim 1.88 \times 10^{4} \mathrm{x}\right)$ magnification using what might be called a Coulomb lens.

\section{Concluding remarks}

It should not be possible for any known particle to create the type of smooth elliptical tracks in photographic emulsion shown in Fig. 2, 3, 4, 5, and 10 and the ranges of possible central forces suggests the elimination of all but magnetic force as the cause of these tracks.

Using a geometrical analysis and analogy with the electron, these particles are shown to possess a (relativistic) mass of $1.45 \times 10^{-3} \mathrm{eV} / \mathrm{c}^{2}$ and a superluminal velocity. A related yet independent analysis [20] based on the Schwinger quantization condition shows that due to the size of the orbits these particles must possess superluminal velocities and a mass of $1.45 \times 10^{-3} \mathrm{eV} / \mathrm{c}^{2}$. Using a new extended relativity model, the experimental results appear to be consistent with the detection of superluminal electrons with $m_{0}=5.11 \times 10^{5} \mathrm{eV} / \mathrm{c}^{2}$, but more work needs to be done to determine conclusively what exactly is producing these tracks.

A new model builds upon The Dirac/Schwinger quantization condition, Recami and Mignani's extended theory of relativity and Coulomb's law to bring a simple superluminal scale transformation presumably without imaginary quantities in the Coulomb flip. Distortion observed by subluminal observers may well be that of a uniform scale change and not a shape change as a result of a translation. Detection of superluminal objects may open a path to substantial magnification of submicroscopic objects analogous to, but with a much greater magnification than, gravitational lensing for astronomical objects.

\section{Acknowledgments}

The author thanks Jacques Consiglio for essential help and stimulating discussions and is also grateful to Moses Fayngold, Giovanni Modanese and Leonid Urutskoev for important suggestions and interest in this work.

\section{References}

[1] L. Epele, H. Fanchiotti, C. García Canal, and V. Vento, Eur. Phys. J. C 56, 87 (2008).

[2] K. A. Fredericks, Eng. Phys. 6, 15 (2013).

[3] C. F. Powell, The study of elementary particles by the photographic method (Pergamon Press, London, 1959); W. H. Barkas, Nuclear Research Emulsions: Vol 1:Techniques and 
theory (Academic Press, New York, 1963); W. H. Barkas, Nuclear Research Emulsions: Vol 2: Particle Behavior and Emulsion Applications (Academic Press, New York, 1973).

[4] L. I. Urutskoev, V. I. Liksonov, and V. G. Tsinoev, Ann. Fond. L. de Broglie 27, 701 (2002), arXiv:physics/0101089 .

[5] L. I. Urutskoev, Ann. Fond. L. de Broglie 29, 1149 (2004).

[6] D. Priem, C. Daviau, and G. Racineux, Ann. Fond. L. de Broglie 34, 103 (2009).

[7] C. Daviau, D. Fargue, D. Priem, and G. Racineux, Annales de la Fondation Louis de Broglie 38, 139 (2013).

[8] N. G. Ivoilov, Ann. Fond. L. de Broglie 31, 115 (2006).

[9] B. Rodionov and I. Savvatimova, in Proceedings of the 12th International Conference on Cold Fusion, edited by A. Takahashi, K. Ota, and Y. Iwamura (World Scientific, 2005) Chap. 44, pp. 421-429; I. Savvatimova and J. Dash, in The 9th International Conference on Cold Fusion, Condensed Matter Nuclear Science. 2002. Tsinghua Univ., Beijing, China, edited by Z. Z. Li (Tsinghua Univ. Press, 2002).

[10] S. V. Adamenko and V. I. Vysotskii, in Proceedings of the 14th International Conference on Condensed Matter Nuclear Science and the 14th International Conference on Cold Fusion (ICCF-14) 10-15 August 2008 Washington DC, edited by D. J. Nagel and M. E. Melich (New Energy Foundation, Inc., 2008) p. 484; S. V. Adamenko and V. I. Vysotskii, Ann. Fond. L. de Broglie 33, 13 (2008).

[11] V. A. Skvortsov and N. I. Vogel, in Exotic Nuclei: EXON-2014 - Proceedings of International Symposium., edited by Y. Penionzhkevich and Y. Sobolev (World Scientific Publishing Co. Pte. Ltd., 2015) pp. 457-463.

[12] H. D. Ables, AAS Photo-Bull. 3, 18 (1971); T. A. Babcock, AAS Photo-Bull. 13, 6 (1976).

[13] T. H. James, The Theory of the photographic process, Fourth Ed. (Macmillan, New York, 1977).

[14] R. P. Czwakiel and E. C. Scott, Proc. SPIE 0058, 192 (1975).

[15] G. Kaye, T. Laby, and N. P. L. G. Britain), Tables of Physical \& Chemical Constants (16th edition 1995). 2.7.7 Cosmic Rays, Kaye \& Laby Online. Version 1.0 (2005) (National Physical Laboratory, 2005).

[16] C. Dilworth, S. Goldsack, Y. Goldschmidt-Clermont, and F. Levy, Philos. Mag. 41, 1032 (1950); I. Barbour, Phys. Rev. 78, 518 (1950); M. Merlin, Nuovo Cimento 11, 218 (1954). 
[17] V. Lebedev and I. Beigman, in Physics of Highly Excited Atoms and Ions (Springer Berlin Heidelberg, 1998) pp. 1-9.

[18] P. A. M. Dirac, Proc. Royal Soc. A 133, 60 (1931); P. A. M. Dirac, Phys. Rev. 74, 817 (1948).

[19] J. Schwinger, Phys. Rev. 144, 1087 (1966).

[20] K. A. Fredericks, Phys. Essays 30, 269 (2017).

[21] T. Datta, Lett. Nuovo Cimento 37, 51 (1983).

[22] H. C. Corben, Int. J. Theor. Phys. 15, 703 (1976).

[23] E. Recami and R. Mignani, Nuovo Cimento 4, 209 (1974); E. Recami, Nuovo Cimento 9, 1 (1986).

[24] L. Parker, Phys. Rev. 188, 2287 (1969).

[25] A. Barut, G. Maccarrone, and E. Recami, Nuovo Cimento A 71, 509 (1982).

[26] B. S. Rajput, P. Joshi, and O. P. S. Negi, Lett. Nuovo Cimento 35, 147 (1982).

[27] H. C. Corben, Lett. Nuovo Cimento 11, 533 (1974).

[28] L. Nottale, Found. Sci. 15, 101 (2010).

[29] R. Mignani and E. Recami, Lett. Nuovo Cimento 9, 367 (1974).

[30] E. Recami and R. Mignani, Lett. Nuovo Cimento 9, 479 (1974).

[31] K. A. Fredericks, J. Condens. Matter Nucl. Sci. 15, 203 (2015).

[32] G. Lochak, Z. Naturforsch. A62, 231 (2007), arXiv:0801.2752 [quant-ph] .

[33] G. Lochak, in The Leptonic Magnetic Monopole Theory and Experiments, Advances in Imaging and Electron Physics, Vol. 189, edited by P. W. Hawkes (Elsevier, 2015) pp. 1 -172 .

[34] V. A. Skvortsov and N. I. Vogel, in Exotic Nuclei: EXON-2014 - Proceedings of International Symposium. Edited by PENIONZHKEVICH YURI \& SOBOLEV YURI. Published by World Scientific Publishing Co. Pte. Ltd., 2015. ISBN \#9789814699464, pp. 465-470, edited by Y. Penionzhkevich and Y. Sobolev (2015) pp. 465-470.

[35] Y. P. Terletskii, Paradoxes in the Theory of Relativity (Plenum, New York, 1968) pp. 102-104. 
[36] V. A. Skvortsov and N. I. Vogel, in in Proceedings of the 29th EPS Conference on Plasma Phys., ECA 26B, D-5.013 (2002).

[37] N. G. Ivoilov and L. I. Urutskoev, Ann. Fond. L. de Broglie 29, 1177 (2004).

[38] G. Bardout, G. Lochak, and D. Fargueb, Ann. Fond. L. de Broglie 32, 551 (2007).

[39] D. Priem et al., Ann. Fond. L. de Broglie 33, 129 (2008).

[40] H. Stumpf, in The Leptonic Magnetic Monopole Theory and Experiments, Advances in Imaging and Electron Physics, Vol. 189, edited by P. W. Hawkes (Elsevier, 2015) pp. 173 -314 . 